\title{
DEUTERIUM ABUNDANCE IN THE LOCAL ISM
}

\author{
JEFFREY L. LINSKY AND BRIAN E. WOOD \\ JILA, Univ. of Colorado and NIST, Boulder, CO 80309-0440 \\ $U S A$
}

\begin{abstract}
Excellent HST/GHRS spectra of interstellar hydrogen and deuterium Lyman- $\alpha$ absorption toward nearby stars allow us to identify systematic errors that have plagued earlier work and to measure accurate values of the $\mathrm{D} / \mathrm{H}$ ratio in local interstellar gas. Analysis of 12 sightlines through the Local Interstellar Cloud leads to a mean value of $\mathrm{D} / \mathrm{H}=$ $(1.50 \pm 0.10) \times 10^{-5}$ with all data points lying within $\pm 1 \sigma$ of the mean. Deciding whether or not the $\mathrm{D} / \mathrm{H}$ ratio has different values elsewhere in the Galaxy and beyond will be one of the major objectives of the FUSE mission.
\end{abstract}

An accurate measurement of $(\mathrm{D} / \mathrm{H})_{\mathrm{LISM}}$, the $\mathrm{D} / \mathrm{H}$ abundance ratio in the local interstellar medium (LISM), and an assessment of spatial variations of $\mathrm{D} / \mathrm{H}$ in the Galaxy are required to address two critically important questions. First, the largest credible $\mathrm{D} / \mathrm{H}$ ratio in our Galaxy will provide a lower limit to the primordial $\mathrm{D} / \mathrm{H}$ ratio, $(\mathrm{D} / \mathrm{H})_{\text {prim }}$, which constrains the critical density of baryons, $\Omega_{\mathrm{B}}$, present in both luminous and "dark" forms. Second, $(D / H)_{\text {LISM }}$ is the end result of an incompletely understood complex set of chemical evolution processes in the Galaxy. Comparison of the $(\mathrm{D} / \mathrm{H})_{\text {LISM }}$ ratio with $\mathrm{D} / \mathrm{H}$ ratios characteristic of the protosolar nebula and in interstellar gas located elsewhere in the Galactic disk and halo will test our understanding of stellar evolution, stellar mass loss, interstellar physics, and the rate of infall and chemical composition of halo gas.

An accurate value of $(\mathrm{D} / \mathrm{H})_{\text {LISM }}$ has great importance as careful analyses of absorption spectra toward QSO 1937-1009 and QSO 1009+2956 indicate that $(\mathrm{D} / \mathrm{H})_{\text {prim }}=(2.4 \pm 0.3) \times 10^{-5}$ (Tytler et al. 1996; Tytler \& Burles 1996). Thus $(\mathrm{D} / \mathrm{H})_{\mathrm{LISM}}$ is likely much closer to $(\mathrm{D} / \mathrm{H})_{\text {prim }}$ than some authors had thought, and our understanding of Galactic chemical evolution will be tested by a measurement of the small difference (perhaps only a factor of 1.5$)$ between $(\mathrm{D} / \mathrm{H})_{\text {prim }}$ and $(\mathrm{D} / \mathrm{H})_{\mathrm{LISM}}$. 
Lyman line absorption is generally recognized as the most reliable technique for inferring the present value of $\mathrm{D} / \mathrm{H}$ in our local environment. This is a consequence of the Sun being surrounded by a cloud of warm, partially ionized gas called the Local Interstellar Cloud (LIC) (e.g., Lallement et al. 1995; Wood \& Linsky 1997). At the 7,000 $\mathrm{K}$ gas temperature in the LIC (Linsky et al. 1993; 1995), ionization and grain adsorption is nearly the same for $H$ and $D$ and there are very few molecules. Lyman- $\alpha$ absorption by $\mathrm{H}$ and $\mathrm{D}$ can be observed with high $\mathrm{S} / \mathrm{N}$ in HST/GHRS spectra toward nearby stars, provided the hydrogen column density is not so large $\left(N_{H I}<10^{18.7} \mathrm{~cm}^{-2}\right)$ as to obliterate the D line only $81 \mathrm{~km} \mathrm{~s}^{-1}$ away.

Ferlet et al. (1996) reviewed D/H measurements obtained primarily with the Copernicus and IUE spectrographs. These pre-HST studies of Lyman line absorption left a confused picture in which the large range of permitted values of $\mathrm{D} / \mathrm{H}$ for each $\mathrm{LOS}$ is consistent with $\mathrm{D} / \mathrm{H}$ spatial variations of a factor of 2 or larger on very short spatial scales. The flood of beautiful new GHRS spectra has changed this picture dramatically. The first clear indication of this paradigm shift was the measurement of $\mathrm{D} / \mathrm{H}=\left(1.60_{-0.19}^{+0.14}\right) \times 10^{-5}$ for the Capella LOS (Linsky et al. 1993, 1995). Since this result lies outside of the published error bars for all of the previous results for this LOS, we believe that the older results at least for the late-type stars are unreliable because of systematic errors.

GHRS echelle spectra have far higher $\mathrm{S} / \mathrm{N}$ and resolution $\left(3.6 \mathrm{~km} \mathrm{~s}^{-1}\right)$ than Copernicus $\left(15 \mathrm{~km} \mathrm{~s}^{-1}\right)$ and IUE $\left(25-30 \mathrm{~km} \mathrm{~s}^{-1}\right)$ spectra. Since the core of the Lyman- $\alpha$ line is very saturated (optical depths of $10^{5}-10^{6}$ ) and is located on the flat part of the curve of growth, high $\mathrm{S} / \mathrm{N}$ and spectral resolution are critical for inferring $\mathrm{H}$ column densities from the steep outer edges of the core absorption profile. Even with the best available GHRS spectra, however, the column densities are usually more uncertain for $\mathrm{H}$ than D. Accurate fits to the outer edges of the core absorption alone may explain much of the previous scatter in the $\mathrm{D} / \mathrm{H}$ values. We have tried to minimize the following sources of systematic errors:

- GHRS spectra of the Mg II lines and ultra-high resolution spectra of the $\mathrm{Na} \mathrm{I}$ and $\mathrm{Ca}$ II resonance lines typically show many closely spaced narrow velocity components even for short LOS (e.g., Welty et al. 1996). We include all known velocity components in our analysis of the $\mathrm{H}$ and $\mathrm{D}$ lines, but the relative column densities in these components may not be the same as for the metal lines, even for O I and $\mathrm{N}$ I which have nearly the same ionization potential as $\mathrm{H} \mathrm{I}$.

- In their analysis of the very short $(1.3 \mathrm{pc})$ LOS to $\alpha$ Cen $\mathrm{A}$ and $\alpha$ Cen B, Linsky and Wood (1996) found additional absorption on the red side of the Lyman- $\alpha$ line, which they identified as absorption by hot hydrogen in the so-called "hydrogen wall" when the interstellar gas 


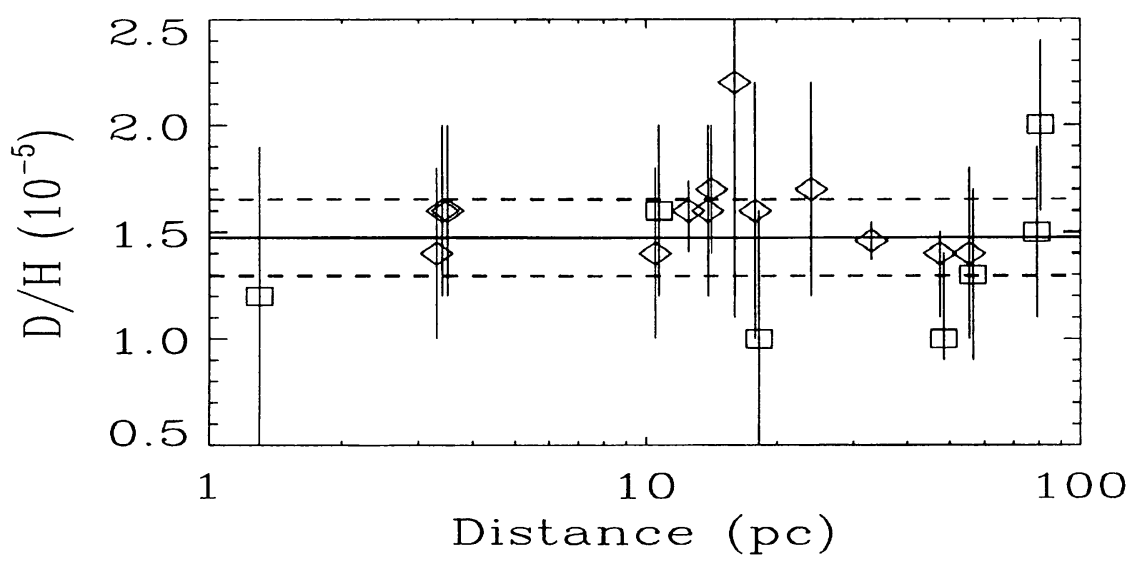

Figure 1. D/H ratios for interstellar gas toward all nearby stars observed with the GHRS. Diamond symbols are for gas in the LIC and square symbols are for other warm clouds. The solid and dashed horizontal lines represent the mean value of $\mathrm{D} / \mathrm{H}$ and the $\pm 1 \sigma$ error of the mean for all data points.

interactes with the solar wind near the heliopause. Subsequent analysis of GHRS spectra of $\epsilon$ Ind, $\epsilon$ Eri, 61 Cyg A, and 40 Eri A identified additional blue absorption in the Lyman- $\alpha$ line produced by hydrogen walls around these stars (Wood, Alexander, \& Linsky 1996; Dring et al. 1997; Wood \& Linsky 1998). If these additional absorption components were not included, then the inferred $\mathrm{H}$ column densities can be a factor of 2 too large and $\mathrm{D} / \mathrm{H}$ a factor of 2 too small.

- The uncertain stellar Lyman- $\alpha$ emission line profile is the "continuum" against which one measures interstellar absorption. One method for minimizing this uncertainty is to analyze spectroscopic binary systems observed at opposite quadratures (when the orbital radial velocities are a maximum) so that the combined stellar emission line profile has a different shape in the two observations. This allows one to infer the intrinsic stellar emission line profiles and the interstellar absorption nearly independent of each other. Linsky et al. (1995) and Piskunov et al. (1997) have used this approach to analyze the LOS toward Capella and HR 1099, and Dring et al. (1997) have used a similar approach to study the LOS toward $\sigma$ Gem. High radial velocity stars allow one to derive $N_{\mathrm{H}}$ I more accurately from the optically thin line wings than from the saturated core (c.f., Wood et al. 1996).

Table I lists all of the published $\mathrm{D} / \mathrm{H}$ values derived for different clouds along the LOS toward nearby stars with GHRS observations. Quantities in 
parenthesis are assumed rather than derived and are not included in the subsequent analysis. Figure 1 shows the derived $\mathrm{D} / \mathrm{H}$ ratios for 12 stars with interstellar radial velocities indicating that their LOS pass through the Local Interstellar Cloud (L) and 7 stars with LOS that pass through other nearby warm clouds ( $G$ refers to the cloud in the Galactic Center direction, NGP refers to the cloud toward the North Galactic Pole, and numbers indicate how many other clouds are in the LOS). The mean value for the LIC is $(\mathrm{D} / \mathrm{H})=(1.50 \pm 0.10) \times 10^{-5}$ and the $\pm 1 \sigma$ error bars for all 12 data points are consistent with the mean value. The horizontal and dashed lines in Figure 4 show the mean relation for all data points, $(D / H)$ $=(1.47 \pm 0.18) \times 10^{-5}$. This figure shows that there is no trend in the $\mathrm{D} / \mathrm{H}$ ratios with distance, and there is no trend with Galactic longitude.

The LIC data indicate that the value of $\mathrm{D} / \mathrm{H}$ in the tiny region of the Galaxy occupied by the LIC is constant and reasonably well known, but we are just beginning to sample more distant lines of sight which may show different $\mathrm{D} / \mathrm{H}$ ratios. The data for the other clouds are more scattered with $(\mathrm{D} / \mathrm{H})=(1.28 \pm 0.36) \times 10^{-5}$. Whether this scatter represents measurement errors or real $\mathrm{D} / \mathrm{H}$ differences between the LIC and the other clouds can only be answered with more high quality observations obtained with FUSE and STIS. FUSE, which is scheduled for launch in October 1998, will observe the less opaque high Lyman lines and thus permit us to study $\mathrm{D} / \mathrm{H}$ further out in the Galactic disk, in the Galactic halo, and toward AGNs.

This work is supported by NASA through grant S-56460-D.

\section{References}

Bertin, P., et al.: 1995, A\&A, 302, 889.

Dring, et al.: 1997, Ap.J., in press.

Ferlet, R. et al. in Science with the HST-II, ed. B. Benvenuti et al. (Space Telescope Science Institute, Baltimore, 1996), p. 450.

Gry, C., et al.: 1995, $A 8 A, 302,497$.

Lallement, R., et al.: 1994, $A \mathcal{E} A, \mathbf{2 8 6}, 898$.

Lallement, R., et al.: 1995, $A \& A, 304,461$.

Landsman, W., Sofia, U.J., \& Bergeron, P.: 1996, in Science with the HST-II, ed. B. Benvenuti et al. (Space Telescope Science Institute, Baltimore), p. 454.

Lemoine, M., et al:: 1996, $A \& A, 308,601$.

Linsky, J. L., et al.: 1993, Ap.J., 402, 694.

Linsky, J. L., et al.: 1995, Ap.J., 451, 335.

Linsky, J. L. \& Wood, B. E.: 1996, Ap.J., 463, 254.

Piskunov, N., et al.: 1997, Ap.J., 474, 315.

Tytler, D., Fan, X.-M., \& Burles, S.: 1996, Nature, 381, 207.

Tytler, D. \& Burles, S.: 1996, in Origin of Matter and Evolution of Galaxies, eds. T. Kajino, Y. Yoshii, \& S. Kubono (World Scientific Publ. Co.)

Welty, D. et al.: 1996, Ap.J. Suppl. 106, 533.

Wood, B.E. \& Linsky, J.L.: 1997, Ap.J, 474, L39.

Wood, B.E. \& Linsky, J.L.: 1998, Ap.J, to appear 10 Jan.

Wood, B. E., Alexander, W. R., \& Linsky, J. L.: 1996, Ap.J., 470, 1157. 
TABLE 1. Summary of GHRS Observations of the LISM.

\begin{tabular}{|c|c|c|c|c|c|c|c|}
\hline \multirow[t]{2}{*}{ Star } & \multirow{2}{*}{$\begin{array}{c}d \\
(\mathrm{pc})\end{array}$} & \multirow{2}{*}{$\begin{array}{c}l \\
\left({ }^{\circ}\right)\end{array}$} & \multirow{2}{*}{$\begin{array}{c}b \\
\left({ }^{\circ}\right)\end{array}$} & \multirow[t]{2}{*}{ Grating $^{\dagger}$} & \multirow{2}{*}{$\begin{array}{l}\text { Clouds } \\
\text { in LOS }\end{array}$} & \multicolumn{2}{|c|}{$\mathrm{D} / \mathrm{H}\left(10^{-5}\right)$} \\
\hline & & & & & & (in LIC) & (others) \\
\hline$\alpha \operatorname{Cen} A^{*}[1,2]$ & 1.3 & 316 & -01 & EA, EB & $\mathrm{G}$ & & $1.2 \pm 0.7$ \\
\hline$\alpha$ Cen B [2] & 1.3 & 316 & -01 & EA, EB & G & & $1.2 \pm 0.7$ \\
\hline Sirius $[1,3,4]$ & 2.7 & 227 & -09 & $\mathrm{~EB}, \mathrm{M}$ & $\mathrm{L}+1$ & $(1.65)$ & $(1.65)$ \\
\hline$\epsilon$ Eri $[11]$ & 3.3 & 196 & -48 & EA, EB & $\mathrm{L}$ & $1.4 \pm 0.4$ & \\
\hline$\epsilon$ Ind [5] & 3.4 & 336 & -48 & EA, EB & $\mathrm{G} / \mathrm{L}$ & $1.6 \pm 0.4$ & \\
\hline Procyon [6] & 3.5 & 214 & +13 & $\mathrm{~EB}, \mathrm{M}$ & $\mathrm{L}+1$ & $1.6 \pm 0.4$ & \\
\hline$\alpha$ Aql [1] & 5.0 & 48 & -09 & EB & $\mathrm{L}+2$ & & \\
\hline$\alpha \operatorname{PsA}[1]$ & 6.7 & 21 & -65 & EB & & & \\
\hline Vega $[1]$ & 7.5 & 68 & +19 & EB & $\mathrm{L}+2$ & & \\
\hline$\beta \mathrm{Gem}[11]$ & 10.6 & 192 & +23 & $\mathrm{EA}, \mathrm{EB}$ & $\mathrm{L}+1$ & $1.4 \pm 0.4$ & $1.6 \pm 0.4$ \\
\hline$\beta$ Leo [1] & 12.2 & 251 & +71 & EB & $\mathrm{L}+2$ & & \\
\hline Capella* $[6,7]$ & 12.5 & 163 & +05 & $\mathrm{EA}, \mathrm{EB}, \mathrm{M}$ & $\mathrm{L}$ & $1.60_{-0.19}^{+0.14}$ & \\
\hline$\beta$ Cas [8] & 14 & 118 & -03 & $\mathrm{M}$ & $\mathrm{L}$ & $1.6 \pm 0.4$ & \\
\hline [11] & & & & & & $1.7 \pm 0.3$ & \\
\hline$\beta$ Cet [8] & 16 & 111 & -81 & $\mathrm{~EB}, \mathrm{M}$ & 2 & $2.2 \pm 1.1$ & \\
\hline$\beta \mathrm{Pic}[1]$ & 16.5 & 258 & -31 & EB & $\mathrm{L}$ & & \\
\hline$\alpha \operatorname{Tri}[11]$ & 18 & 139 & -31 & EA, EB & $\mathrm{L}$ & $1.6 \pm 0.6$ & $1.0 \pm 0.6$ \\
\hline$\lambda$ And [5] & 24 & 110 & -15 & EA & $\mathrm{L}$ & $1.7 \pm 0.5$ & \\
\hline$\delta$ Cas [1] & 27 & 127 & -02 & $\mathrm{~EB}$ & $\mathrm{~L}$ & & \\
\hline HR1099* [8] & 33 & 185 & -41 & $\mathrm{EA}, \mathrm{EB}, \mathrm{M}$ & $\mathrm{L}+2$ & $1.46 \pm 0.09$ & \\
\hline G191-B2B [9] & 48 & 156 & +07 & $\mathrm{~EB}, \mathrm{M}$ & $\mathrm{L}+2$ & $1.4_{-0.3}^{+0.1}$ & (1.),(1.5) \\
\hline$\sigma \mathrm{Gem}^{*}[11]$ & 56 & 191 & +23 & EA, EB & $\mathrm{L}+1$ & $1.4 \pm 0.4$ & $1.3 \pm 0.4$ \\
\hline $\mathrm{HZ} 43$ [12] & 63 & 054 & +84 & EA, EB & NGP & & (1.6) \\
\hline 31 Com [8] & 80 & 115 & +89 & $\mathrm{~EB}, \mathrm{M}$ & NGP & & $1.5 \pm 0.4$ \\
\hline$[11]$ & & & & & & & $2.0 \pm 0.4$ \\
\hline$\epsilon \mathrm{CMa}[10]$ & 187 & 240 & -11 & $\mathrm{~EB}, \mathrm{M}$ & $\mathrm{L}+5$ & $(1.65)$ & \\
\hline
\end{tabular}

Quantities in parenthesis are assumed $\mathrm{D} / \mathrm{H}$ values that lead to good profile fits.

* These stars were observed twice. Capella, HR 1099 and $\sigma$ Gem were observed near opposite quadratures.

$\dagger$ Gratings: $\mathrm{EA}=$ Echelle-A, EB = Echelle-B, M = G140M or G160M.

References: [1] Lallement et al. (1995), [2] Linsky \& Wood (1996), [3] Lallement et al. (1994), [4] Bertin et al. (1995), [5] Wood et al. (1996), [6] Linsky et al. (1995), [7] Linsky et al. (1993), [8] Piskunov et al. (1997), [9] Lemoine et al. (1996), [10] Gry et al. (1995), [11] Dring et al. (1997), [12] Landsman et al. (1996). 